\title{
The Effect of Pull Power of Karaoke Television-Set Service on Eliminating the Internal Psychological Obstacles of Consumer
}

\author{
Jiun-Hung Lin \\ Department of Marketing and Logistics Management, China University of Technology, Hsin-Chu County \\ Email: linjinghung.tem89g@nctu.edu.tw, jiun.hunglin@msa.hinet.net
}

Received May 2014

\begin{abstract}
Since the recreation activities is more diversified because of information technology and that the leisure policy of government provide many free recreation place and activities, KTV service as a kind of recreation activities but not free faces a keener competition in Taiwan. This study tries to explore how to decrease or eliminate obstacles for consumer to choose KTV as their recreation activity through understanding the internal psychological obstacles for KTV consumption with empirical data and provides suggestions to help improve the KTV service.
\end{abstract}

Keywords

Motivation, Karaoke TV (KTV)

\section{Introduction}

Since 1990, KTV is an important recreation activity in Taiwan [1]. Karaoke television-set (KTV) is a form of interactive musical entertainment service provides independent private room and interactive musical facilities for consumer to sing a song with friends or family [2].

However, that the arise of internet and handy smart phone and the constructions of many green and free public parks and places by local government which hold many festival activities attract people to participate and consume, leads to the recreation activities more and more diversified. Hence, as a kind of recreation activities, KTV doesn't face keener competition easily.

Individual may have "internal psychological obstacles" for KTV consumption due to past bad experience, knowledge, personality or personal ability. That how to decrease or eliminate internal psychological obstacles for consumer to choose KTV as their recreation by improving the KTV service factors to help attract consumer who occasionally choose KTV as their recreation option and then expand the market and revenue becomes an important issue to be studied. 


\section{Theoretical Background}

\subsection{Karaoke TV (KTV)}

KTV is originated from Karaoke which comes from Japan [2]. Karaoke which means singing in a public stage with the accompaniment of an orchestra without anybody is introduced to Taiwan in the early 1980. Karaoke needs consumer with courage to sing in the public. However, it's not the case for everyone. Hence, the popularity of Karaoke is limited [3]. Business in Taiwan developed a new type of recreation service to combine Karaoke with an independent and private room and is called as Karaoke television-set (KTV) in the early 1990 [2]. KTV service provides an independent and private room as a singing place for consumer to sing with a vocal accompaniment video tape broadcasted on a larger television and stereo facility. Beverage and meal is also provided for consumer [4].

\subsection{Obstacles of Leisure Activity}

Psychologists indicate motivation is the drive of an individual behavior and is highly related to what people talk and behave [5]. There are positive and negative motivations that can induce individual behavior reaction [5]-[7]. "Positive motivation" means an inner drive that would guide individual behavior while "negative motivation" would resist individual behavior [5] [6]. Negative motivations induced by past bad experience, knowledge, shy personality, or poor personal ability are internal psychological obstacles that will resist consumer to do leisure activity.

The characteristics of the service itself that would attract consumer such as having special atmosphere, exciting facilities, or convenient location are commonly positive motivations and are the pull power for recreations activities [5] [6].

KTV is a "heterologous space for experiencing imagination" and "the great decoration of the independent and private room [8]." Zhang [8] indicates that the living room like design of the KTV room provides a place for consumer to have and share fun with friends. The KTV room is an isolated, safety, and private place and can make a small group abandon themselves to sing songs without any disturbance. The songs called for singing, the stars that perform the songs, and the vocal accompaniment video tape in the KTV room all can be the topic to talk and induce pleasant feeling of interpersonal relationship. Accordingly, the stateroom of KTV leads to stronger and closer interpersonal relationship among people in the room.

For KTV consumption, individual may have internal psychological obstacles to go to KTV because of their past bad KTV consumption experience, shy personality, poor singing skill or knowledge and information about KTV service comes from media or friends. In order to understand how to decrease or eliminate internal psychological obstacles of consumer to KTV, exploring the effect of pull power of KTV service on the internal psychological obstacles of consumer is an important issue to be studied to help attract more and more potential consumer who occasionally go to KTV service and then expand the market and revenue.

\section{Research Model and Methodology}

Based on the discussions in previous sections, this research develop hypothesis to be empirical tested as below.

H1: Pull power of KTV service is negatively related to the internal psychological obstacles of consumer.

A pilot qualitative study through five focus group interviews with twelve members for each focus group interview were designed for exploring the internal psychological obstacles resist consumer go to KTV.

Pull power of KTV service is measured through measurement scales developed by previous related study [9]. Internal psychological obstacles of consumer are measured through measurement scales developed by this study. Likert seven point scale from very disagree to very agree is used to measure all items. Factor analysis is used to extract the factors of pull power of KTV service and internal psychological obstacles of consumer for KTV service. Hierarchical clustering was used to cluster individual by internal psychological obstacles of consumer.

All the questionnaires were answered by the KTV consumer in Taiwan. Regression is used to validate the assumptions developed.

\section{Result}

Four hundred questionnaires were delivered and 227 valid questionnaires received, representing an effective re- 
sponse rate of $57 \%$.

Results of factor analysis indicated that KMO values of both pull power of KTV service and internal psychological obstacles of consumer for KTV service are above 0.85 and Bartlett's Test of Sphericity for each construct is also significant at p-value $<0.01$.

Four factors are extracted for pull power of KTV service and 68.7\% of variance is extracted. Those factors are "V11: Nice general services setting and quality", "V12: Located in urban flourishing business district", "V13: Wonderful singing related facilities", and "V14: Located in recreation and entertainment district".

Two factors are extracted for internal psychological obstacles of consumer to KTV and 61.5\% of variance is extracted. These two factors are "V21: Singing hardware and software is bad" and "V22: Low self-confidence to singing in KTV”.

Consumers whose average scores in one or two of the internal psychological obstacles is larger than four are treated as consumer who has internal psychological obstacles to KTV. Only the "having internal psychological obstacles to KTV" data are selected and used to do regression analysis in this study. The proportion of "having internal psychological obstacles to KTV group” is $61 \%$.

Hierarchical clustering is used to cluster "having internal psychological obstacles to KTV group”. "Having bad KTV experience with high self-confidence to singing (41\%)” and "Having slightly bad KTV experience with low self-confidence to singing in KTV (59\%)” are two groups analyzed.

The regression analysis result indicates the standardized path coefficients and $R^{2}$ for each regression function in this study is presented in Figure 1.

For "having bad KTV experience with high self-confidence to singing group", the $R^{2}$ values for "Singing hardware and software is bad" and "low self-confidence to singing” are 0.47 and 0.03 , respectively. "Nice general services setting and quality" is not significantly related to both "Singing hardware and software is bad" and "Low self-confidence to singing". "Located in urban flourishing business district" is not significantly related to both "Singing hardware and software is bad" and "Low self-confidence to singing". "Wonderful singing related facilities" is significantly negatively related to "Singing hardware and software is bad" but not significantly related to "Low self-confidence to singing". "Located in recreation and entertainment district" is significantly positively related to "Singing hardware and software is bad" but not significantly related to "Low self-confidence to singing”.

For "having slightly bad KTV experience with low self-confidence to singing in KTV group", the $R^{2}$ values for "Singing hardware and software is bad" and "low self-confidence to singing" are 0.13 and 0.14 , respectively. "Nice general services setting and quality" is not significantly related to both "Singing hardware and software is bad" and "Low self-confidence to singing". "Located in urban flourishing business district" is not significantly related to both "Singing hardware and software is bad" and "Low self-confidence to singing”. "Wonderful singing related facilities" is significantly negatively related to both "Singing hardware and software is bad" and "Low self-confidence to singing”. "Located in recreation and entertainment district” is not significantly related to both "Singing hardware and software is bad" and "Low self-confidence to singing".

Having bad KTV experience with high self-confidence to singing group Having slightly bad KTV experience with low self-confidence to singing group

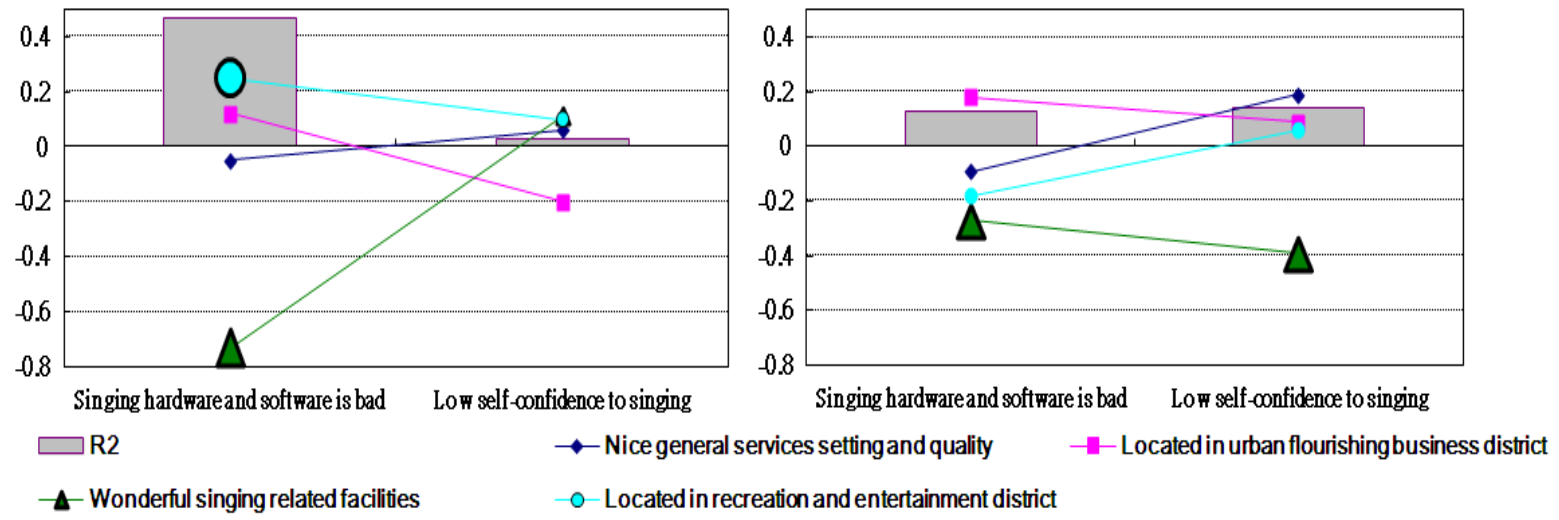

Figure 1. Results of theoretical model. ${ }^{*}$ Big bold point denotes a significant path $(\mathrm{p}<0.01)$. 


\section{Conclusion and Discussion}

Based on the research result of this research, the internal psychological obstacles of consumer to KTV would build obstacles to resist consumer to KTV. The pull power is induced by the KTV service itself. The two main internal psychological obstacles for consumer to KTV are "Singing hardware and software is bad" and "Low self-confidence to singing”. The four main pull powers induced by the characteristics of the KTV service itself are "nice general services setting and quality”, "located in urban flourishing business district”, "wonderful singing related facilities", and "located in recreation and entertainment district". Some of the pull powers induced by the service itself would help decrease the internal psychological obstacles of consumer to KTV.

Consumer who have internal psychological obstacle due to past bad KTV service consumption experience induced by bad singing hardware and software is the main internal psychological obstacles to KTV while low self-confidence to singing before the crowd is secondary psychological obstacle. Two types of customers who have internal psychological obstacles to KTV are identified.

For consumer who has internal psychological obstacles to KTV because of having bad KTV experience but with high self-confidence to singing, KTV services should try their best to provide wonderful singing related facilities in each KTV room but avoid setting the KTV located in recreation and entertainment district.

For consumer who has internal psychological obstacles to KTV because of having slightly bad KTV experience with low self-confidence to singing, KTV stores should try their best to improve singing related facilities in each KTV room. Moreover, KTV stores should try their best to develop singing related facilities and system that can help these consumers sing songs better.

Furthermore, for attracting consumer who has internal psychological obstacles to KTV, KTV services are suggested to set KTV stores located in urban flourishing business district. The general hardware and service outside the KTV room such as the lobby setting is suggested to be set in an acceptable level as the average industry standard.

\section{References}

[1] Chiang, Y.Z. (2006) One Million Lonely Heart Wait for Happiness in the Three Metro City. Global Views Magazine, 238, 166-174.

[2] Sha, G.G. (1992) The Politic Economic of Recreation-The First Analysis to the KTV in Taiwan. Journal of Outdoor Recreation Study, 5, 145-163.

[3] Su, F.S. (2006) The Study of Motivations, Obstructions, and Health Effects of Teenage Students to Sing at KTV Stores. Unpublished Master Thesis of Department of Leisure and Recreation, Asia University, Taichung.

[4] DCAMEA (Dept of Commercial Affairs of Ministry of Economic Affairs) (2000) The Change and Future Trend of Audio-Visual Entertainment Industry. Annual Report of Service Business in Taiwan, 21-46.

[5] Fodness, D. (1994) Measuring Tourist Motivation. Annals of Tourism Research, 21, 555-581.

[6] Gnoth, J. (1997) Tourism Motivation and Expectation Formation. Annals of Tourism Research, 24, $283-304$. http://dx.doi.org/10.1016/S0160-7383(97)80002-3

[7] Chang, Z.S. (1990) Chang’s Psychology Dictionary. Tunghua Publish Ltd., Taipei.

[8] Zhang, S.M. (1993) Leisure, Pleasure, and Power-A Sociological Analysis of KTV in Taiwan. Unpublished Master Thesis of Graduate Institute of Building and Planning, National Taiwan University, Taipei.

[9] Lin, J.H. (2013) The Effect of Pull Power of Karaoke Television-Set Service on the Inner Need of Consumer. iBusiness, 5, 52-54. 